\title{
Decay of finite temperature superfluid helium-4 turbulence
}

\author{
Demosthenes Kivotides \\ Department of Aeronautics, Imperial College London, \\ London SW7 2AZ, United Kingdom
}

(Dated: June 3, 2015)

\begin{abstract}
A mesoscopic model of superfluid helium-4, that describes the dynamics of individual topological defects of the ground state (superfluid vortices) and their (self-consistent) interactions with its quasi-particle excitations (normal-fluid), is solved numerically in order to analyse the physics of decaying homogeneous, isotropic turbulence. The calculations predict several temporal decay regimes not present in classical turbulence decay, the corresponding superfluid and normal-fluid energy spectra, and the experimentally observed $t^{-1.5}$ scaling for the superfluid-vortex line density at large times. The results demonstrate that the origin of this scaling is the energy spent by the superfluid in order to sustain a fluctuating low Reynolds number flow in the normal-fluid, and not the locking of turbulent superfluid and normal-fluid vorticities.
\end{abstract}

PACS numbers: 


\section{PROLOGUE}

The spontaneous breaking of global U(1) symmetry in $\mathrm{He}^{4}$ (Bose-Einstein Condensation, in short BEC) results in a ground (vacuum) state with nonzero field expectation value, that can be treated as a Schroedinger coherent state [1]. The hydrodynamic, Nambu-Goldstone mode [2] that results from the spontaneous symmetry breaking of the ground state is is the phase of the mean field, and the corresponding, conserved, Noether current of the $\mathrm{U}(1)$ symmetry of the purely low-energy physics (Goldstone mode) Lagrangian is the superfluid momentum (phase-field gradients). Similar ideas apply also to classical fluids (e.g., breaking of rotational symmerty in nematic liquid crystals [3]), but BEC He is special, since the Goldstone mode is an inviscid fluid, distinct from the normal-fluid of Bogolyubov's quasi-particle excitations of the ground state. Under suitable excitation, the superfluid can develop a complicated tangle of topological defects (vortices) of universal circulation (superfluid turbulence). Hence, in finite temperature, $\mathrm{BEC} \mathrm{He}^{4}$ flows, two very different kinds of turbulence (classical and superfluid) can coexist in constant interaction with each other.

This intriguing interplay between two highly nonlinear phenomena has been the topic of many experimental and theoretical investigations [4-10], that indicated the fecund spatial spectral structure of turbulence in superfluid $\mathrm{He}^{4}$. Indeed, Kivotides has computed directly from a mesoscopic, self-consistent model of superfluid hydrodynamics the spectral structure of forced superfluid turbulence $[9,10]$. There are three scaling regimes in both the normalfluid and superfluid. In the normal-fluid [10], there is a low $k$ Kolmogorov $k^{-5 / 3}$ regime, a high $k, k^{-2.2}$ regime that corresponds to the a creeping flow sustained via energy input from the superfluid vortices, and an intermediate $k^{-6}$ transition regime. In the superfluid, there is a low $k, k^{-5 / 3}$ regime, that is due to the large scale organization of the superfluid tangle by the normal-fluid turbulence eddies, an intermediate $k^{-3}$ scaling that corresponds to the growth phase of superfluid vorticity due to its interaction with intense vorticity structures in the normal-fluid [9], and a high $k, k^{-1}$ regime that corresponds to the probing of individual linevortices. The Kelvin waves cascade appears to be damped [10] even at the relatively small temperature of $T=1.3 \mathrm{~K}[13]$. Here, I focus instead on the temporal aspects of decaying turbulence, by analysing the pioneering, homogeneous, isotropic turbulence experiment of 

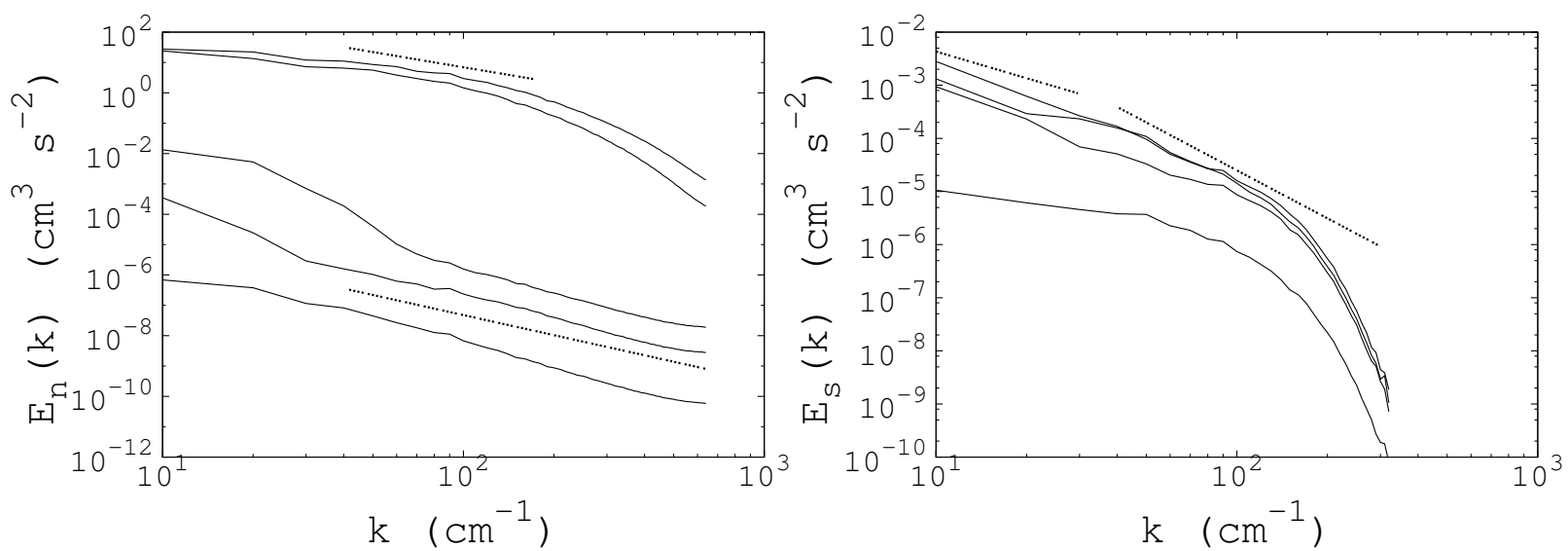

FIG. 1: Left: Normal-fluid energy spectra. From top to bottom, the times are: $t=0, t=0.021 \mathrm{~s}$, $t=0.061 \mathrm{~s}, t=0.25 \mathrm{~s}$ and $t=2.021 \mathrm{~s}$. The top dotted straight line signifies the $k^{-5 / 3} \mathrm{scaling}$, and the bottom one the $k^{-2.2}$ scaling. The small extension of the $k^{-5 / 3}$ regime in the initial data is due to the relatively small $R e_{\lambda}$ value. In agreement with the spatial spectra of [10], the initial Kolmogorov regime is eliminated due to energy losses, and replaced by a low Reynolds number, viscosity-dominated $k^{-2.2}$ regime. Right: Superfluid energy spectra. From top to bottom, the times are: $t=0.021 \mathrm{~s}, t=0.061 \mathrm{~s}, t=0.25 \mathrm{~s}$ and $t=2.021 \mathrm{~s}$. The two dotted straight lines signify the $k^{-5 / 3}$ and $k^{-3}$ scalings. In agreement with the spatial spectra of [10], a fully developed vortex tangle is characterized by a low wavenumber $k^{-5 / 3}$ scaling regime, and an adjacent $k^{-3}$ range. The scalings disappear later on, when $\kappa \Lambda$ becomes very small (bottom curve).

[5]. In agreement with experiment, mesoscopic theory predicts the $t^{-1.5}$ scaling [5] for the superfluid-vortex line density at large times, indicating further a sequence of temporal-decay regimes which are not present in classical turbulence, and correspond to different modes of interaction between normal-fluid and superfluid turbulence. A key finding of the present study is that the energetics of superfluid turbulence decay have not been understood correctly in the past. Indeed, a terminal $t^{-1.5}$ scaling regime in the decay of vortex line density was interpreted as a turbulent scaling (attributed to the locking of inertial range normal-fluid and superfluid vorticities), when it actually corresponds to a low Reynolds number fluctuating flow in the normal-fluid, that is sustained by continuous energy transfer from the superfluid (at the expense of superfluid vortex length). 


\section{MESOSCOPIC THEORY OF FINITE TEMPERATURE SUPERFLUIDS}

This work employs the mesoscopic model of superfluid physics developed in [10]. As the term "mesoscopic" implies, this model is valid in the range of scales between the microscopic and macroscopic regimes. By miscroscopic, I mean here the range of scales described by atomic quantum field theory [11]. Within this theory, the superfluid vortex core structure is fully resolved at nanoscale distances, and the quasi-particle excitations of the vacuum state are described directly at an individual particle level. The macroscopic description is obtained by fully coarse graining the microscopic degrees of freedom. In this way, both superfluid vorticity and quasi-particle excitations appear as a continuum. This is the conceptual framework of the statistical closures of the Hall-Vinen and Gorter-Mellink type, and the vortex dynamical reformulation of the former by Schwarz [12]. The key point here is that writing statistical closures for superfluid vorticity (and the normal-fluid/superfluid coupling) is not an easier task than the analogous (unsolved) problem of classical turbulence, hence, the usefulness of existing macroscopic equations as purely predictive theories of superfluid physics is a delicate matter (as is also in classical turbulence). It is for this reason that the mesoscopic model is proposed: it refers to an intermediate range of scales, where the quasi-particle excitations form a continuum fluid, but the superfluid vortices remain discrete, hence their dynamics are explicitly described, and no coarse grained modeling is required. A key advantage of the mesoscopic description is the explicit capturing of topological change via reconnections, and its dynamical impact. Moreover, crucial experimental information such as superfluid line vortex densities can only be obtained via a mesoscopic approach and is not available in macroscopic formulations. Notably, the superfluid vortex dynamics are partially coarse-grained in this description, hence there is no vortex core resolution, and reconnections appear as jump processes. Finally, it is important to note that the present formulation is not a full-blown mesoscopic description; it applies only in the limit of incompressible and isothermal flow processes.

In BEC quantum fluids [10], a tangle of superfluid vortices $\mathcal{L}$ interact with a normal-fluid 
of velocity $\mathbf{V}_{n}$. The motion of a vortex point $\mathbf{X}_{v}(t)$ is described by

$$
\begin{array}{r}
\mu_{v} \ddot{\mathbf{X}}_{v}+\rho_{s} \kappa \mathbf{X}_{v}^{\prime} \times\left(\mathbf{V}_{s}-\dot{\mathbf{X}}_{v}\right)+\rho_{n} \kappa \mathbf{X}_{v}^{\prime} \times\left(\mathbf{V}_{n}-\dot{\mathbf{X}}_{v}\right)+ \\
D_{0} \mathbf{X}_{v}^{\prime} \times\left[\mathbf{X}_{v}^{\prime} \times\left(\mathbf{V}_{n}-\dot{\mathbf{X}}_{v}\right)\right]-g \dot{\mathbf{W}}- \\
\int_{-\infty}^{+\infty} d t^{\prime}\left[\int_{\mathcal{L}-V_{\epsilon}\left(\mathbf{X}_{v}\right)} d\left|\mathbf{X}_{\mathcal{L}}\right| \mu_{v} \ddot{\mathbf{N}}_{R} \delta\left(\left|\mathbf{X}_{v}-\mathbf{X}_{\mathcal{L}}\right|\right)\right] \delta\left(t-t^{\prime}\right)=0 .
\end{array}
$$

Here, $\mu_{v}$ is the vortex mass per unit length, $\mathbf{X}_{v}^{\prime}$ the unit tangent to the tangle, $\rho_{s}$ the superfluid mass density, $\kappa$ the quantum of circulation, $\rho_{n}$ the normal-fluid mass density, $D_{0}$ the Hall-Vinen drag coefficient, $\mathbf{V}_{s}$ the Biot-Savart velocity $\mathbf{V}_{s}\left(\mathbf{X}_{v}\right)=\frac{\kappa}{4 \pi} \int_{\mathcal{L}} \frac{\left(\mathbf{x}-\mathbf{X}_{v}\right) \times d \mathbf{x}}{\left|\mathbf{x}-\mathbf{X}_{v}\right|^{3}}, g \dot{\mathbf{W}}$ a white noise process $[10,14]$ (the derivative of the Wiener process $\mathbf{W}$ ), and $\mathbf{N}_{R}$ a deterministic jump process that models the transition from one smooth superfluid tangle configuration to another. If $V_{\epsilon}^{-}(\mathbf{X})$ is the neighborhood of radius $\epsilon \rightarrow 0$ of point $\mathbf{X}$ along vortex tangle $\mathcal{L}$ in the direction of smaller arc-length values (against the vorticity direction), and similarly for $V_{\epsilon}^{+}(\mathbf{X})$, then $\mathbf{N}_{R}$ describes the topological jump $\left(V_{\epsilon}^{-}\left(\mathbf{X}_{v}\right) \cup V_{\epsilon}^{+}\left(\mathbf{X}_{v}\right)\right) \oplus\left(V_{\epsilon}^{-}\left(\mathbf{X}_{\mathcal{L}}\right) \cup V_{\epsilon}^{+}\left(\mathbf{X}_{\mathcal{L}}\right)\right) \rightarrow$ $\left.\left(V_{\epsilon}^{-}\left(\mathbf{X}_{v}\right) \cup V_{\epsilon}^{+}\left(\mathbf{X}_{\mathcal{L}}\right)\right) \oplus\left(V_{\epsilon}^{-}\left(\mathbf{X}_{\mathcal{L}}\right) \cup V_{\epsilon}^{+}\left(\mathbf{X}_{v}\right)\right)\right)$. The requirement of smooth post-jump configuration entails the dissipative nature of vortex reconnections in superfluids [10]. The other forces in the equation are (from start to end): vortex inertia, Magnus, Iordanskii lift, Hall-Vinen (mutual-friction) drag, and thermal fluctuation forces. The components $f_{F} \equiv g \dot{W}$ of the thermal fluctuations force at any location on the vortex tangle are Gaussian stochastic variables with mean value zero, and time-correlator $<f_{F}\left(t_{1}\right) f_{F}\left(t_{2}\right)>=2 D_{0}\left(k_{B} T / \ell_{F}\right) \delta\left(t_{1}-t_{2}\right)$, where $\ell_{F}$ could be taken to be the length scale of the numerical discretization along the vortices. The normal-fluid obeys standard Navier-Stokes dynamics, i.e., the mass equation $\nabla \cdot \mathbf{V}_{n}=0$, and the momentum equation

$$
\begin{aligned}
& \frac{\partial \mathbf{V}_{n}(\mathbf{x}, t)}{\partial t}+\nabla\left(\frac{p}{\rho_{n}+\rho_{s}}+\frac{\mathbf{V}_{n} \cdot \mathbf{V}_{n}}{2}\right)-\mathbf{V}_{n} \times\left(\nabla \times \mathbf{V}_{n}\right)- \\
& \frac{\mu}{\rho_{n}} \nabla^{2} \mathbf{V}_{n}-\kappa \int_{\mathcal{L}} d\left|\mathbf{X}_{\mathcal{L}}\right|\left[\mathbf{X}_{\mathcal{L}}^{\prime} \times\left(\mathbf{V}_{n}-\dot{\mathbf{X}}_{\mathcal{L}}\right)\right] \delta^{3}\left(\mathbf{x}-\mathbf{X}_{\mathcal{L}}\right)- \\
& \frac{D_{0}}{\rho_{n}} \int_{\mathcal{L}} d\left|\mathbf{X}_{\mathcal{L}}\right|\left\{\mathbf{X}_{\mathcal{L}}^{\prime} \times\left[\mathbf{X}_{\mathcal{L}}^{\prime} \times\left(\mathbf{V}_{n}-\dot{\mathbf{X}}_{\mathcal{L}}\right)\right]\right\} \delta^{3}\left(\mathbf{x}-\mathbf{X}_{\mathcal{L}}\right)=0
\end{aligned}
$$

Here, $p$ is the pressure field, and $\mu$ the normal-fluid viscosity. From start to end, we have the inertia, potential ("Bernoulli-group"), vortex, viscous, lift and drag (mutual-friction) forces [10]. The last two forces signify the coupling of normal-fluid with superfluid vortices, and will be collectively called "vortex couplings". Notably, there are two energy sinks in the system: (a) vortex reconnections for superfluid kinetic energy, and (b) viscous dissipation 
for normal-fluid kinetic energy. The numerical methodology and corresponding computational algorithms are discussed in reference [10], which also includes information regarding the numerical implementation of the "reconnection-force", and normal-fluid, grid mesh-size requirements for well resolved turbulence calculations. In the present results, thermal fluctuation effects on the vortices are neglected, and the employed numerical scheme does not resolve vortex inertial relaxation processes, since, due to very small vortex mass densities, the latter are too fast to be of relevance in the time-scales of interest [10]. For the vortices, I renormalize the self-interaction velocity divergence in the Biot-Savart law by employing the velocity of a ring with radius the local radius of curvature, and apply the method of Winckelmans and Leonard for evaluating velocity contributions because of all other points [15]. For the latter, an effective vortex core radius equal to $\Delta \xi$ is employed [15].

\section{COMPUTATIONAL MODELING}

In the experiment of [5], a homogeneous, isotropic turbulent flow was created at $T=1.5 \mathrm{~K}$, by towing a grid through a stationary sample of superfluid $\mathrm{He}^{4}$. Following the passage of the grid, the vortex line density $\Lambda=L / \mathcal{V}$, where $L$ is the superfluid vortex length, and $\mathcal{V}$ the system volume, with actual experimental value $\mathcal{V}=(1 \mathrm{~cm})^{3}$, was measured. The $\kappa \Lambda$ results showed a monotonic $\Lambda$ decrease, obeying a $t^{-1.5}$ scaling law for long decay times.

In order to model these computationally, I have chosen $T=1.3 \mathrm{~K}$, and a (periodic) computational box of volume $\mathcal{V}=(0.1 \mathrm{~cm})^{3}$. For this temperature, the various constants have the following values: $\rho_{s}=0.13860 \mathrm{~g} \mathrm{~cm}^{-3}, \rho_{n}=0.00652 \mathrm{~g} \mathrm{~cm}^{-3}, D_{0} / \rho_{n}=0.905 \times 10^{-3} \mathrm{~cm}^{2} \mathrm{~s}^{-1}$, and $\nu=2.330 \times 10^{-3} \mathrm{~cm}^{2} \mathrm{~s}^{-1}$. Notably, since $\kappa=0.997 \times 10^{-3} \mathrm{~cm}^{2} \mathrm{~s}^{-1}$, all three basic parameters, i.e., $D_{0} / \rho_{n}, \nu$ and $\kappa$ has similar values. Experimental grid-towing, and the creation of homogeneous, isotropic turbulence in both fluids is modeled as follows: (a) I start with an initial, Gaussian, small Reynolds number, normal-fluid velocity field in the classical (no vortex couplings) Navier-Stokes equation, (b) I apply a Lundgren (so called linear) forcing [10], and evolve the pure normal-fluid to a turbulent steady-state characterized by Taylor Reynolds number $R e_{\lambda} \approx 55$, and Kolmogorov's $k^{-5 / 3}$ energy scaling over approximately a 

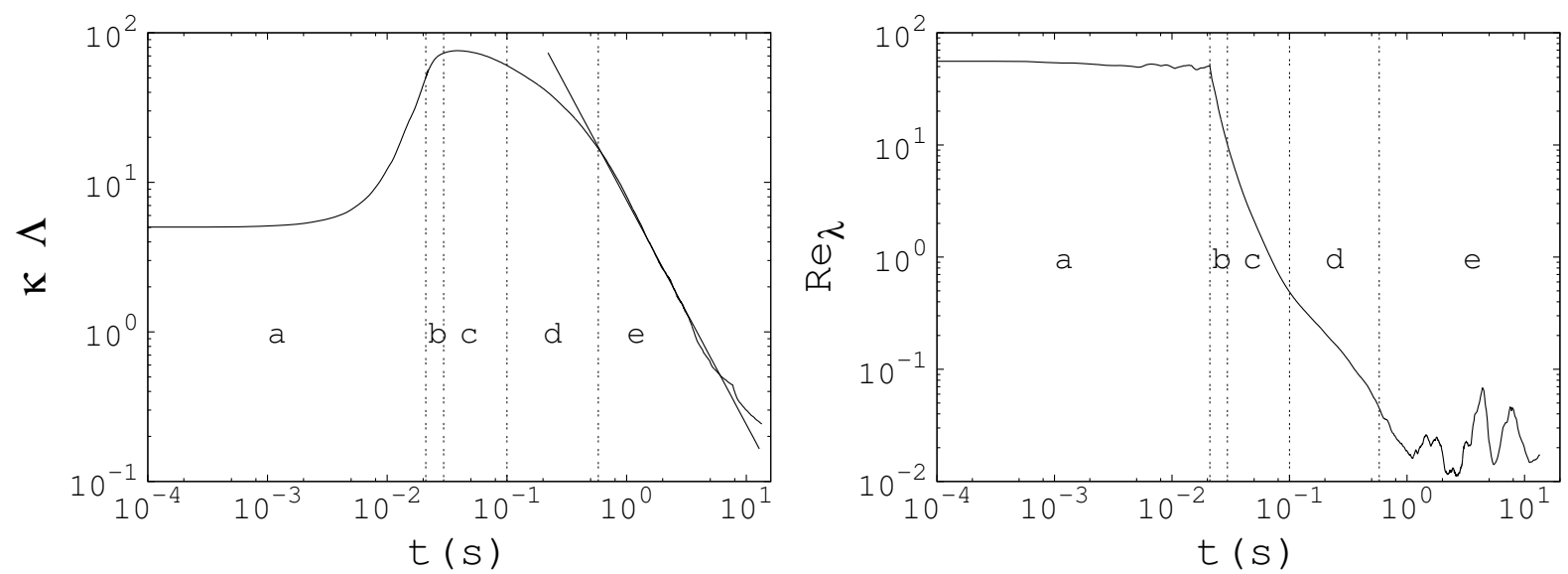

FIG. 2: Left: $\kappa \Lambda$ versus time. The four dotted vertical lines are indicative of (1) the end of the initial forcing period $(t=0.021 \mathrm{~s}),(2)$ the ceasing of important inertial effects in the normal-fluid $\left(R e_{\lambda}=10\right.$ when $\left.t=0.0296 \mathrm{~s}\right),(3)$ the end of an approximate plateau in vortex line density at $t=0.1 \mathrm{~s}$, and (4) the end of an intermediate regime of $\Lambda$ decay between the above mentioned plateau and the start of the final scaling regime $(t=0.58 \mathrm{~s})$. The straight line indicates the experimentally measured $\kappa \Lambda \propto t^{-1.5}$ scaling law, that the mesoscopic model predicts. I have also indicated (a-e) the five decay regimes discussed in the text. Right: $R e_{\lambda}$ versus time. The four dotted vertical lines (at same times as in $\kappa \Lambda$ graph) are indicative of (1) the end of the initial forcing period, (2) the bulk of normal-fluid turbulence decay (which is the analogous process here to classical turbulence decay), (3) the end of the $R e_{\lambda}$ decay range that corresponds to the approximate $\kappa \Lambda$ plateau, (4) the end of a slower $R e_{\lambda}$ reduction rate period, and the beginning of a (fluctuating) equilibrium regime, where the normal-fluid acts like a low Reynolds number "bath" of quasi-particle excitations. The equilibrium is sustained via energy transfer from the superfluid to the normal-fluid that compensates viscous dissipation.

decade in wavenumber space (Fig. 1, left, top curve); notably, the somewhat small $k$-range exhibiting the inertial scaling, is due to the, computational complexity constrained, not too large $R e_{\lambda}$ value, (c) I add to the above prepared, pure normal-fluid turbulence, a set of 40 randomly-oriented/positioned, seed, superfluid vortex rings with randomly distributed radii between $r_{\max }=0.25 l_{b}$ and $r_{\min }=0.75 r_{\max }=0.1875 l_{b}$ (where $l_{b}$ is the computational box size), so that $\kappa \Lambda \approx 5 \mathrm{~s}^{-1}$, and I employ the combined flow system as initial conditions in the present, fully coupled calculation ( $t=0$ in the graphs), (d) as a theoretical analog of exper- 

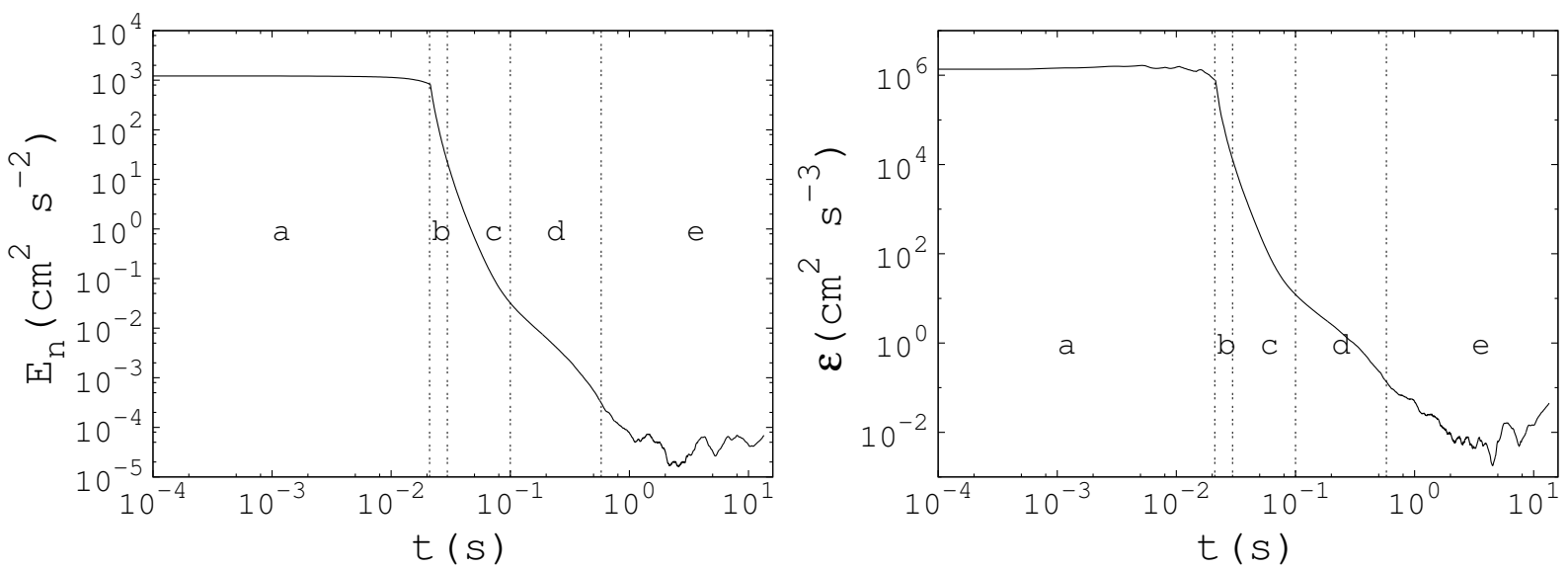

FIG. 3: Left: Normal-fluid energy $E_{n}$ versus time $t$. Right: Viscous energy dissipation rate $\epsilon$ versus time $t$. The four vertical lines correspond to the same times as in Fig. 2. Both $E_{n}$ and $\epsilon$ dynamics show similarities with $R e_{\lambda}$ evolution. This appears reasonable, since the latter scales with the third power of the turbulence intensity (i.e., with $E_{n}^{3 / 2}$ ), and $\epsilon$ depends on the derivatives of the velocity field, which decrease during decay following the loss of flow inertia.

imental grid-towing, I continue forcing the normal-fluid during the initial phase of the fully coupled calculation, in order to (also) generate a homogeneous, isotropic turbulence state in the superfluid. The latter is achieved via the combined action of energy transfer to the vortex tangle from the normal-fluid (via drag and lift forces, i.e., vortex couplings), and the reconnection-force, i.e., the jump process $\mathbf{N}_{R}$ that creates "chaotic" vortex configurations via topological changes, and the accompanying Kelvin-wave excitations. Discontinuation of linear forcing, that models the end of grid-towing in the experiment, occurs after the initial transient of turbulence build-up in the superfluid, at $t=0.021 \mathrm{~s}$. At this time, due to energy losses to the superfluid, the Taylor Reynolds number in the normal-fluid has dropped to $R e_{\lambda} \approx 50$, whilst Kolmogorov's $k^{-5 / 3}$ scaling is still discernible over approximately a decade in wavenumber space (Fig. 1 , left), and $\kappa \Lambda$ has increased from its seed value to $\kappa \Lambda \approx 51 \mathrm{~s}^{-1}$ (Fig. 2, left). Subsequently, the unforced turbulence in the BEC quantum fluid (i.e., superfluid plus normal-fluid) is allowed to decay due to the combined action of superfluid vortex reconnection and viscous dissipation processes. 


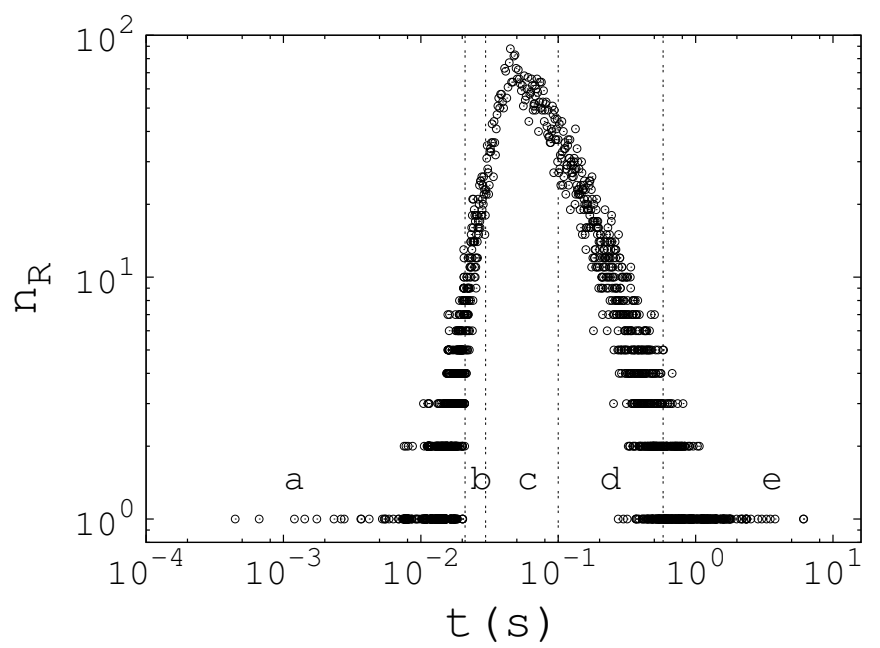

FIG. 4: Number of tangle reconnections $n_{R}$ versus time $t$. The four vertical lines correspond to the same times as in Fig. 2. Notably, the $\kappa \Lambda \propto t^{-1.5}$ scaling (regime e) corresponds to the decay of a very dilute vortex tangle, with negligible reconnection induced dynamical effects, that sustains a very low energy bath of quasi-particle excitations.

\section{PHYSICS OF FINITE TEMPERATURE SUPERFLUID TURBULENCE DECAY}

Next, I discuss the stages of quantum-fluid turbulence decay that the mesoscopic model predicts. Starting from the normal-fluid, there are five temporal decay regimes, exemplified here by following the evolution of $R e_{\lambda}=u^{\prime} \lambda / \nu$ (where the Taylor microscale $\lambda=15 \nu\left(u^{\prime}\right)^{2} / \epsilon$, and $\epsilon$ is the viscous energy dissipation rate) versus time (Fig. 2, right): (a) an initial, forced turbulence regime (ending at $t=0.021 \mathrm{~s}$ ), where $R e_{\lambda}$ decreases very slowly, because viscous losses are fully compensated by the linear forcing (analog of grid-towing), and normal-fluid kinetic energy losses are solely due to vortex couplings with the superfluid, which, as demonstrated in [10], are much weaker than viscous effects, (b) an (unforced) stage of strong viscous dissipation of turbulence emergy, and weaker energy transfer to the vortex tangle, that ends at $t=0.0296 \mathrm{~s}$, when inertial effects in the normal-fluid have drastically subsided (here, by convention, this is taken to imply $R e_{\lambda}<10$ ); indeed, as shown in (Fig. 1, left), and in agreement with [10], during this period, the inertial range is replaced by the dissipative $k^{-2.2}$ scaling that prevails thereafter, (c) a period of (relatively) fast, $R e_{\lambda} \propto t^{-1.4}$, low Reynolds number, normal-fluid flow decay (ending at $t=0.1 \mathrm{~s}$ ), (d) a stage of slower, $R e_{\lambda} \propto t^{-1}$, low Reynolds number, normal-fluid flow decay (ending at $t=0.58 \mathrm{~s}$ ), and (e) a final period, where the normal-fluid energy has reached an equilibrium (albeit fluctuating) 
level, that is sustained via energy input from the superfluid. The latter is necessarily the case, since if the transfer of energy had the inverse direction, there would have been two energy sinks in the normal-fluid, hence a monotonic energy decrease. Notably, it is not obvious which is the direction of energy transfer during periods (c) and (d). Thus, I have computed directly the average rate of work performed by vortex couplings. The results are better understood in conjunction with $\kappa \Lambda$ dynamics (Fig. 2, left): period (c) corresponds to an approximate plateau of $\kappa \Lambda$; starting from the peak of this plateau, the energy transfer to the superfluid starts diminishing, and towards the end of regime $(\mathrm{c})(t \approx 0.08 \mathrm{~s})$, it reverses direction. Thus, the milder $R e_{\lambda}$ decay rate during phase (d) is due to the fact, that in opposition to phase (c), the normal-fluid is forced by the superfluid during this period.

Correspondingly, there are five temporal evolution regimes for the superfluid, exemplified here by following the change of $\kappa \Lambda$ versus time: (a) an initial $\kappa \Lambda$ build-up regime where vortex couplings transfer energy from the forced normal-fluid turbulence to the tangle; as shown in Fig. 1 (right), and in agreement with [10], the superfluid energy spectrum presents evidence of a low wavenumber $k^{-5 / 3}$ scaling range, followed by a steeper $k^{-3}$ scaling range at higher wavenumbers, (b) a subsequent, short-lived stage corresponding to the diminishing of inertial effects in the normal-fluid (hence, also, of its potential for energy transfer to the superfluid); during this period, superfluid kinetic energy keeps growing, because vortex-coupling effects, that act as energy sources, overpower vortex reconnection effects that act as energy sinks, (c) a time period corresponding to the (relatively) fast decay of a low-Reynolds number normal-fluid; here, $\kappa \Lambda$ presents a plateau, and remains approximately constant, as a gradual reversal of the energy transfer direction from the superfluid to the normal-fluid takes place in the system, (d) a stage corresponding to the slower decay of a low-Reynolds number normal-fluid, during which $\kappa \Lambda$ decays faster in comparison with phase (c), since, as discussed above, during this period, the superfluid is characterized by two energy sinks: both vortex reconnections and vortex couplings, (e) a $\kappa \Lambda \propto t^{-1.5}$ scaling regime that is observed in the experiments; during this stage, results for the rate of work performed by vortex couplings, indicate that energy keeps flowing from the superfluid to the normal-fluid. The difference with range $(\mathrm{d})$ is that, here, viscous dissipation is not as strong (after all, the normal-fluid energy is very small), hence vortex couplings can, at times, overpower viscous effects, and increase the normal-fluid energy; the higher levels of the latter, 
in turn, result in higher energy dissipation rates that overpower vortex-coupling induced energy transfer to the normal-fluid, and the cycle repeats itself. Remarkably, as shown in Fig. 1 (right), by this time, the spectral scaling structure of superfluid energy is lost, as the tangle becomes a very dilute system of vortices, that cannot be properly characterized as "turbulent". Notably, the mechanism of Kondaurova and Nemirovskii [16] of vortex line decay due to evaporation (or diffusion) of vortex loops from the bulk is not active here. This is because this mechanism is properly valid for $T \rightarrow 0 \mathrm{~K}$ temperatures, and relies on Kelvin waves generating many small loops in the system. However, for the finite temperature $T=1.3 \mathrm{~K}$ here, the Kelvin waves cascade is damped, and small ring production is negligible.

It is important to note, that [5] have erroneously associated regime (e) with a "locking" of (inertial-range) normal-fluid and superfluid vorticities. This is not supported by the calculation, since during the $t^{-1.5}$ scaling, there are no inertial effects in the normal-fluid (Fig.1, left), and, instead, there exists a fluctuating, creeping flow that, by default, does not posses any concentrated vorticity. A close comparison of theoretical and experimental results indicates that (1) the reported experimental data do not include the superfluid vorticity build-up (i.e., periods (a) and (b) above), and (2) the experimental runs corresponding to the smallest grid velocities, i.e., $5 \mathrm{~cm} / \mathrm{s}$ and $10 \mathrm{~cm} / \mathrm{s}$, are remarkably similar to the theoretical results. Indeed for these cases, there is excellent quantitative agreement between the peak $\kappa \Lambda$ values, as well as the actual times over which the $t^{-1.5}$ scaling is observed. Notably, in both theory and experiment, the $t^{-1.5}$ scaling involves the final decay-times, hence this scaling cannot involve normal-fluid turbulence. Indeed, in the latter case, one would anticipate a transition of this scaling towards a laminar (low Reynolds number) one, but both theory and experiment agree that the $t^{-1.5}$ scaling is a terminal scaling, a fact consistent with the low Reynolds number flow in the normal-fluid predicted by the mesoscopic theory. Notably, the theoretical results for both fluids refer only to turbulent fluctuations. Indeed, by construction, the mean flow in the normal-fluid is identically zero, and the initial superfluid rings have a random orientation. Any mean-flow effects in the experiment must also be small during the time-period of the scaling law, because the superfluid vorticity is decaying. In the absence of vortex locking, the creeping normal-fluid flow during the $t^{-3 / 2}$ scaling indicates that the latter is a purely superfluid turbulence scaling, with a small superfluid energy loss due to the couplings with (an 
otherwise unimportant) normal-fluid. Hence, it is crucial to understand the spectral signature of these couplings (i.e., their strength and distribution across scales) before proceeding further with a scaling theory of the underlying turbulence physics.

Fig. 3 presents the evolutions of normal-fluid energy $E_{n}$ and viscous energy dissipation rate $\epsilon$, whilst tangle reconnections $n_{R}$ are shown in Fig. 4. Evidently, the experimentally observed $\kappa \Lambda \propto t^{-1.5}$ scaling corresponds to the decay of a very dilute tangle without any significant reconnection effects. Not only are the latter very few, at most, one at a time, but also, due to strong Kelvin wave damping [10], their dynamical effects are too weak.

\section{EPILOGUE}

The present calculation provides a detailed picture of the decay of (relatively) low $R e_{\lambda}$, BEC quantum-fluid turbulence. It is expected that the results for stages (c) to (e) would be universally valid for all (initial) $R e_{\lambda}$. This is because the flow phenomenology during these phases does not depend on the initial level of forcing, since the normal-fluid has very small inertia, hence no memory of its initial, energetic turbulent state. Indeed, this is another way of explaining the universality of late-time decay observed in the experiments. However, depending on the ratio of the (turbulence) time-scales of normal-fluid energy decay and superfluid vorticity build-up, potentially interesting new physics could, perhaps, emerge in stages (a)-(b) for much higher $R e_{\lambda}$. Some of them are hinted by the complex-fluid viewpoint of superfluids (as developed in [10]). Moreover, the good agreement between theory and experiment obtained here, builds confidence in the usefulness of the mesoscopic approach, on top of its accurate prediction [17] of particle velocities in the counterflow suspension experiments of [18].

This computation is a first step towards more advanced studies of fine statistical aspects of superfluid turbulence. Key issues such as interscale energy transfer, intermittency, and coherent structures are important physics to be examined in the future. 
[1] A.J. Leggett, Quantum Liquids, Oxford University Press (2006).

[2] D. Forster, Hydrodynamic Fluctuations, Broken Symmetry, and Correlation Functions, W.A. Benjamin, Inc. (1975).

[3] H. Pleiner, H. R. Brand, in Pattern Formation in Liquid Crystals eds. A. Buka and L. Kramer, Springer (1996).

[4] J. Maurer, P. Tabeling, Europhys. Lett. 43, 29 (1998).

[5] S.R. Stalp, L. Skrbek, R.J. Donnelly, Phys. Rev. Lett. 82, 4831 (1999).

[6] W. Guo, J.D. Wright, S.B. Cahn, J.A. Nikkel, D.N. McKinsey, Phys. Rev. Lett. 102, 235301 (2009).

[7] P.M. Walmsley, A.I. Golov, H.E. Hall, A.A. Levchenko, W.F. Vinen, Phys. Rev. Lett. 99, $265302(2007)$

[8] W.F. Vinen, J. Low Temp. Phys. 161, 419 (2010).

[9] D. Kivotides, J. Fluid Mech. 668, 58 (2011).

[10] D. Kivotides, Phys. of Fluids 26, 105105 (2014).

[11] E.A. Calzetta, B-L B. Hu, Nonequilibrium Quantum Field Theory, Cambridge University Press (2008).

[12] K. W. Schwarz, Phys. Rev. B 31, 5782 (1985).

[13] D. Kivotides, Phys. Lett. A 341, 193 (2005).

[14] L. Thompson, P.C.E. Stamp, Phys. Rev. Lett. 108, 184501 (2012).

[15] D. Kivotides, S.L. Wilkin, J. Low Temp. Phys. 156, 163 (2009)

[16] L. Kondaurova, S.K. Nemirovskii, Phys. Rev. B 86, 134506 (2012).

[17] D. Kivotides, Phys. Rev. B 78, 224501 (2008).

[18] T. Zhang, S. W. Van Sciver, J. Low Temp. Phys. 138, 865 (2005). 